\title{
VALIDATION OF A SURVIVABLE PUBLISH-SUBSCRIBE SYSTEM
}

\author{
Sankalp Singh ${ }^{1}$, Adnan Agbaria ${ }^{1}$, Fabrice Stevens ${ }^{2}$, Tod Courtney ${ }^{1}$, \\ John F. Meyer ${ }^{3}$, William H. Sanders ${ }^{1}$, Partha Pal ${ }^{4}$ \\ ${ }^{1}$ University of Illinois at Urbana-Champaign, USA. \{sankalps, adnan, tod,whs\}@crhc.uiuc.edu \\ ${ }^{2}$ France Telecom, France. fabrice.stevens@francetelecom.com \\ ${ }^{3}$ University of Michigan, USA.jfm@eecs.umich.edu \\ ${ }^{4}$ BBN Technologies, Cambridge, MA, USA.ppal@bbn.com
}

\begin{abstract}
We describe, with respect to high-level survivability requirements, the validation of a survivable publishsubscribe system that is under development. We use a top-down approach that methodically breaks the task of validation into manageable tasks, and for each task, applies techniques best suited to its accomplishment. These efforts can be largely independent and use a variety of validation techniques, and the results, which complement and supplement each other, are seamlessly integrated to provide a convincing assurance argument. We also demonstrate the use of model-based validation techniques, as a part of the overall validation procedure, to guide the system 's design by exploring different configurations and evaluating trade-offs.
\end{abstract}

Keywords: Quantitative Validation, Security Verification, Information Assurance, Probabilistic Modeling, Intrusion Tolerance, Security

\section{INTRODUCTION}

The emergence of large distributed information systems to support nation-critical needs (e.g., electrical power distribution, telecommunications, military command and control, and health care) has spurred research into new system protection strategies that can produce a system whose critical function will survive in spite of hostile attacks, complex failures, or accidents [1]. These new strategies have drawn upon traditional approaches such as prevention technologies (e.g., cryptography, authentication, and firewalls), detection technologies (e.g., network sensors and host-based sensors), fault-tolerance technologies (e.g., agreement protocols), and the like, and combined them with newer technologies that enable dynamic defensive responses such as changing security policies or isolating suspected components. Successful strategies interleave these building blocks into a solution that remains cost-effective and manageable even when scaled to very large systems.

The validation of survivable systems, like their construction, must use an integrated approach. In addition to well-known security requirements such as confidentiality and integrity, a survivable system may also need to satisfy probabilistic constraints on its behavior. Such constraints impose different modeling and reasoning strategies. This is particularly true of survivable systems that make use of intrusion tolerance technologies [2, 3, 4], since by definition, intrusion tolerance is a probabilistically quantified property of the system. Moreover, since impairments may manifest themselves in implementation details, we must rely on focused testing to convince ourselves that the implementation is faithful to the modeled design. A successful validation approach must collect this disparate evidence into a single, seamlessly integrated assurance argument that is convincing to the accreditor.

Most traditional approaches to validation of security have been process-based, usually in the form of guidelines [5]. Goal-based evaluations, when attempted, have usually either been based on formal methods [6] and aimed to prove that certain security properties hold given a specified set of assumptions, or been informal, using teams of experts (often called "red teams," e.g., [7]) to try to compromise a system. Quantitative methods, in particular probabilistic modeling, have been receiving increasing attention as a mechanism to validate security. Any probabilistic model intended to validate a secure system would have to represent, among other things, the attacker's behavior. Since 
some of the vulnerabilities in an information system will be unknown at the time the system is designed (and modeled), the prediction of when and how an attacker may successfully intrude the system is a difficult, but critically important problem. Early work on probabilistic validation of secure systems was done by Littlewood et al. [8]. Their work was exploratory in nature and identified "effort" made by an attacker as an appropriate measure of the security of the system. Jonsson and Olovsson [9] attempted to build a quantitative model of attacker behavior using data from several experiments they conducted over a two-year period. They postulated that the process representing an attacker may be broken into multiple phases, each of which has an exponential time distribution. Attempts have been made to build models that take into account behavior of the system as well as the attacker, and the uncertainties therein. Madan et al. [10] have used a semi-Markov model to evaluate the security properties of the SITAR architecture, an intrusiontolerant system. Their model does not explicitly represent the attacker or the vulnerabilities that may lead to intrusions, but represents the state of the system in terms of high-level events that may lead to failures. Sheyner et al. [11] have tried to build attack trees automatically using formal methods, and then analyze those trees using Bayesian networks. Ortalo et al. [12] have proposed modeling of known system vulnerabilities using "privilege graphs," followed by a combination of the privilege graphs with simple assumptions about attacker behavior to obtain "attack-state graphs." The latter can be analyzed using Markov techniques to obtain probabilistic measures of security. Singh et al. [13] have used probabilistic modeling to validate an intrusion-tolerant system, emphasizing the effects of intrusions on the system behavior and the ability of the intrusion-tolerant mechanisms to handle those effects, while using very simple assumptions about the discovery and exploitation of vulnerabilities by the attackers to achieve those intrusions. Gupta et al. [14] have used a similar approach to evaluate the security and performance of several intrusion-tolerant server architectures. In most of the above efforts, the designers chose modeling assumptions and model parameterswithout incorporating the justifications for those choices into a larger validation framework. Moreover, the representation of the attacker's behavior is highly simplified. The chief value of existing research in this area is that it demonstrated the applicability of these approaches as evaluation techniques that can be used as components of an integrated assurance argument.

The safety-critical systems community has been using "safety cases" [15] as a means to express arguments about the guaranteed safety of systems such as nuclear power plants. While safety cases allow disparate kinds of evidence to be incorporated into an overall argument, they primarily serve as a visual aid, and do not give formal guidelines as to how an argument/requirement is decomposed, especially if it is quantitative in nature. The models used for generating actual evidence in argument trees are usually simplistic; there is a lack of traceability between the actual design and the models; and quantitative evidence (typically generated by probabilistic models) is generally in the form of a leaf, with no argument subtree enumerating and justifying the assumptions made in the models. Furthermore, an approach based on an integrated argument has not been applied to validation of security-related properties.

We have recently developed and applied a validation method for survivable systems that includes the use of logical arguments, stochastic simulations, and experiment-based testing with actual system components (e.g., red teaming [7]). The need for such diversity is due, in part, to the basic nature of a survivability requirement, which can generally be decomposed into sub-requirements that differ with regard to the types of techniques that can be applied. We applied the method to a proposed intrusion-tolerant design for a publishsubscribe system (hereafter referred to as IT Pub$\mathrm{Sub}$ ), both to demonstrate that the proposed design satisfies imposed survivability requirements and to help us consider different protection trade-offs as we developed the final design. The resulting assurance argument incorporates each piece of assurance evidence as a supporting argument of some higher-level claim.

A major component of our validation approach was probabilistic modeling. The probabilistic models used an innovative attacker model. The attacker model has a sophisticated and detailed representation of various kinds of effects of intrusions on the behavior of system components (such as a variety of failure modes). It includes a representation of the process of discovery of vulnerabilities (both in the operating system(s) and in the specific applications being used by the system) and their subsequent exploitation, and considers an aggressive spread of attacks through the system by taking into account the connectivity of the components of the system, at both the infrastructure and the logical levels. We believe that this attacker model is applicable to a wide range of secure and intrusion-tolerant systems. Moreover, we demonstrate the use of probabilistic modeling, as embedded in the IVP, to compare different design configurations, allowing the designers of the 
system to make choices that maximize the survivability of the system before it is actually implemented.

The remainder of the paper is organized as follows. Section 2 provides an overview of the publish-subscribe system studied. Section 3 provides the outline of our validation procedure. Section 4 provides the details and results of the validation procedure. Finally, we conclude in Section 5 with a summary of lessons learned and prospects for further evolution of our work.

\section{OVERVIEW OF THE IT PUB-SUB DESIGN}

The IT Pub-Sub system consists of multiple clients communicating with each other through a central core, as shown in Fig. 1. The core consists of three zones (or layers) of components: the crumple zone (outermost layer), the operations zone, and the executive zone (innermost layer). The network connectivity is constrained, through the use of network-interface-level hardware firewalls and configurable network switches, to limit direct network connectivity from the outside network to the inner zones. Communication between machines in each zone is accomplished via proprietary communication protocols.

The primary component in the crumple zone is the access proxy (AP). It functions as a bridge between the outer (public) network containing the clients and the inner core network for the remaining zones. It translates between multiple publicly supported communication interfaces (such as RMI and CORBA) and the single internal core communications protocol. Clients connect to the access proxy to publish, subscribe, and query IO. Attacks on clients generate alerts that are forwarded to the core via the AP, and commands to the client security components from the core pass back to the client via the AP.

Inside the crumple zone is the operations zone. It contains the components that perform the two main functions of the core: processing IO objects, and monitoring and maintaining the security of the core and the clients connected to it. IO processing is performed by three operations zone components: the PSQ server (PSQ), the downstream controller (DC), and the guardian (Gu). The PSQ server receives IO objects sent to the core via the AP in the crumple zone. The DC verifies the signatures on messages sent from clients to ensure data integrity. The guardian uses scenario-specific and domainspecific knowledge to identify corruption within the contents of the IO.

Security monitoring and maintenance are performed by local components distributed across all of the hosts in the client, the crumple zone, the operations zone, and a centralized correlator (Co) in the operations zone. The components local to individual hosts are sensors, actuators, and local controllers (LC). Sensors are dedicated to intrusion detection, actuators are mechanisms that carry out actions when commanded, and an LC is the control agent responsible for local survivability management.

The correlator receives alerts from multiple intrusion detection sensors within the client, crumple, and operations zones, filters out redundant and false alerts, and forwards serious messages to the system manager. The correlator interprets the alerts it receives in the context of the global state of the system, allowing it to better identify redundant alerts and false alarms.

The executive zone contains the system manager (SM). It serves as the master controller of the IT Pub-Sub. It monitors intrusion alerts received from the correlator and generates commands for the appropriate response to counter the intrusion. Human operators monitor the IT Pub-Sub via displays generated by the SM, and can manually initiate specific responses from the SM.

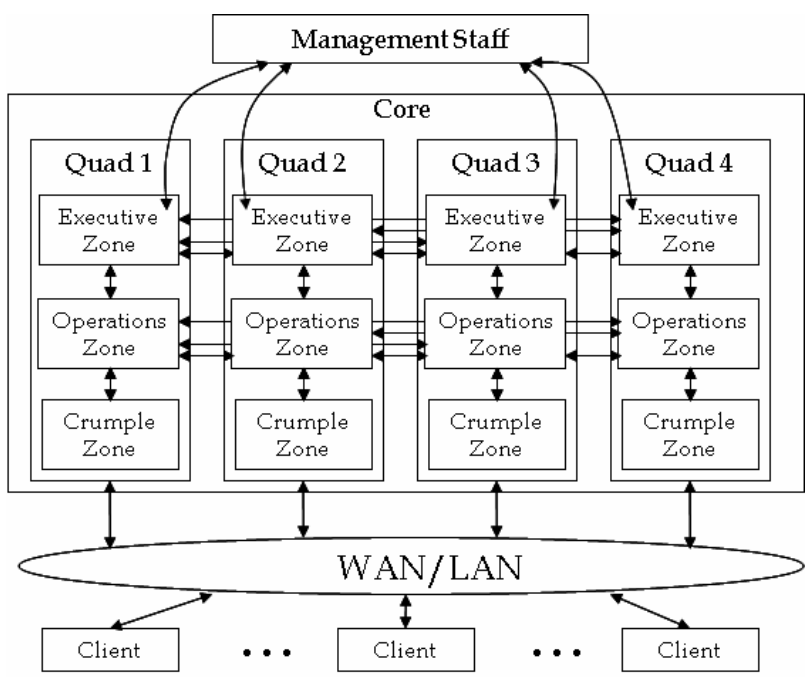

Fig. 1 - The IT Pub-Sub Architecture

The core is redundant, consisting of four quadrants (or quads) that run different operating systems. Each quadrant contains copies of all crumple, operation, and executive zone components discussed previously. Agreement protocols run among the SM and PSQ servers to ensure a common, collective view of the system state by human operators viewing it through the SM displays, and by clients interacting with the core via the access proxies in the crumple zone. The baseline configuration of the system used in our case study assigned the same operating system to all the hosts within a single quadrant of the core. 


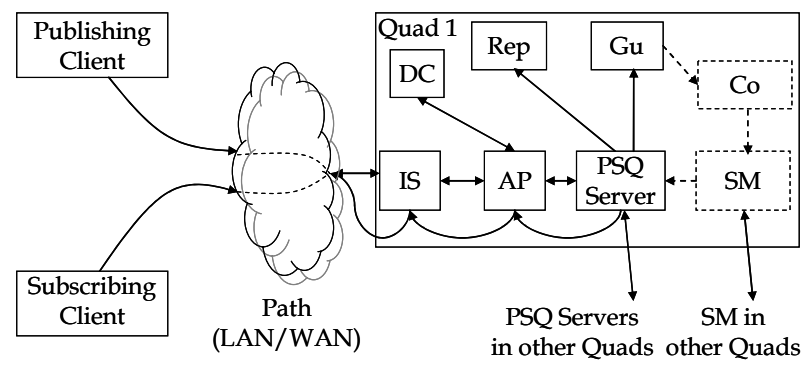

Fig. 2 - Publish Data Flow

Among the various data flows in the system, two are central to the validation effort described in the sections that follow. The first, which is depicted in Fig. 2, is the data flow among the system components during the publish operation. The publish operation begins when a client creates an IO to be published. The IO is signed using the client's private session key and sent to the access proxy in one of the quadrants through the "publish" protocol. The access proxy receives the IO through the isolation switch (IS) and sends it to the DC to verify whether the client is in a valid session. After successful verification, the AP sends the IO to the PSQ server in its quadrant. The PSQ server forwards the IO to the other PSQ servers in the other quadrants. Each PSQ server then stores the IO in its repository (Rep), sends to the client an acknowledgment of the receipt of the publication, and sends the IO to the guardian. The guardian performs domain-specific tests on the IO. If it finds an error in the IO, it sends an alert to the correlator. The correlator determines, based on the threat level of the alert and the alert state of the system, whether the alert is likely to represent an attack. If it is, the alert is forwarded to the SM. The SMs collectively decide whether they should tell the PSQ servers to recall the IO. If there are no recalls from the SM, the IO is accepted, and subscribing clients are notified that a new IO is available. The subscribing clients then query the system for the available IO.

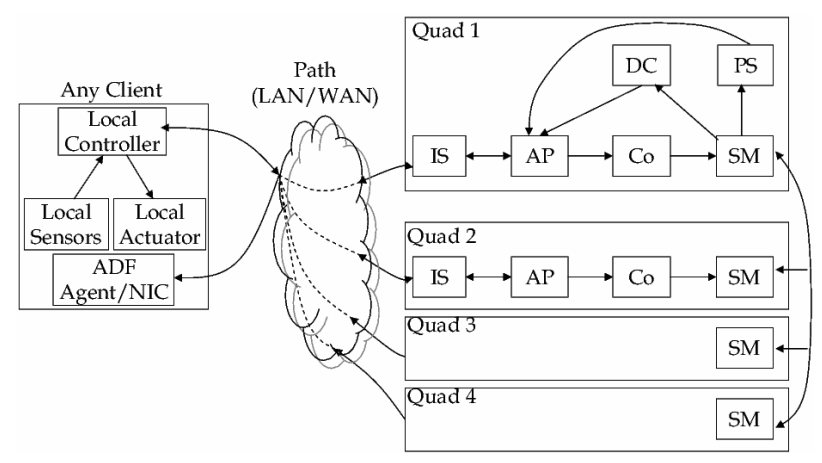

Fig. 3 - Alert/Response Data Flow of a Client

The second data flow of interest is the alert/response data flow associated with the intrusion detection and tolerance capabilities of the system. Alerts are generated on the clients and on components in the core, and the data flow is similar for both cases. The data flow for client alerts is presented in Fig. 3 and described here. When a sensor in a client host detects an anomalous condition/attack, it generates an alert and sends it to the LC on the same host. The LC can either make a local response, such as restarting a process or replacing a corrupted file, or forward the alert to the core for a coordinated adaptive response by the correlator. The correlator then decides (using correlation with other alerts) if this alert is critical, determines the compromised client(s), and informs the SM in its quadrant. The SMs propagate the reports within the SM group and reach a consensus to take action. Each SM then generates a command for the desired response on the client generating the alert and sends it to the client's LC via the DC and AP. The LC commands the appropriate local actuator on the client to take action. If the SM group determines that a more drastic response is appropriate, the client can be quarantined. Each SM notifies the policy server (PS) for the hardwarebased firewall system and isolates the compromised client.

\section{VALIDATION APPROACH}

Before we delve into the the details of the validation, we clarify the nature of high-level survivability requirements and formulate a theoretical basis for their decomposition into subrequirements. We then provide an outline of the procedure we employed to validate the IT Pub-Sub with respect to particular high-level requirements.

\section{Survivability Requirements}

As defined in [1], survivability is the capability of a system to fulfill its mission, in a timely manner, in the presence of attacks, failures, or accidents. A system is survivable if it has the above-stated capability according to a specified set of survivability requirements. The latter are statements that collectively imply what is meant by the system's "capability to fulfill its mission in an adverse operational environment." (In what follows, it is assumed that "in a timely manner" is accounted for as part of "mission fulfillment.") A survivable system's operational environment includes both mission-specific interactions on the part of intended users (its use environment) and adverse interactions due to attacks and faults (the attack/fault environment).

The term "capability" is particularly important in defining survivability requirements. In some instances, it suffices to identify capability with "certainty," i.e., fulfillment occurs with probability 1. However, capability often needs to be otherwise 
quantified, and that often involves probabilities. To illustrate this, let us suppose $X$ is some service that is essential to fulfilling the system's mission. Then a corresponding survivability requirement for the system, call it ExR for "Example Requirement," could be the following.

ExR: Whenever requested, $X$ is successfully delivered with a probability of at least $p_{X}(0$ $<p_{X}<1.0$ ),

where "successfully delivered" needs to be further elaborated in terms relating to both the system and the mission objectives. For example, suppose that $X$ involves the transfer of a data object from one system user to another. Then, successful delivery (provision) of this service, among other things, can depend on the end-to-end data flow required to realize $X$, timeliness of the data flow, and integrity of the received data object, along with other security properties such as authentication (the sender is an authorized user) and confidentiality (the data object is not disclosed to an unauthorized recipient).

Generally, a system requirement can be viewed as a predicate $R(s)$, where the variable $s$ refers to a system along with relevant aspects of its operational environment. When applied to a specific system $\mathrm{S}$, the proposition $R=R(\mathrm{~S})$ is then a requirement for $\mathrm{S}$, where $R$ is satisfied by $\mathbf{S}$ (alternatively, $S$ is valid with respect to $R$ ) if the truth value of $R$ is true. When a requirement $R$ for a particular system is being stated, the system is usually understood from context, and hence not referred to explicitly in the statement of $R$. (See the definition of ExR above, in which we omitted saying "by the system" after "successfully delivered.") This practice will be followed throughout the paper.

With a slight abuse of terminology, we regard a requirement as being quantitative if deciding whether $R$ is satisfied by the system entails the evaluation of at least one quantitative measure. In the case of high-level survivability requirements, such quantification is likely probabilistic, as illustrated in the example given above. This is due mainly to uncertainties in the system's operational environment (i.e., use environment and attack/fault environment) together with defense mechanisms that may behave probabilistically. Evaluation of the corresponding measure(s) can be based on a model of the system and its operational environment, experimentation with an actual system (operating in an actual or simulated environment), or some combination of the two. In addition to measure evaluation, other techniques need to be brought into play to determine whether a quantitative requirement is satisfied. If a requirement is non- quantitative, then its satisfaction can generally be decided without direct invocation of evaluation results for any quantitative measure. (Indirectly, such results can provide evidence that the requirement is indeed non-quantitative.)

Generally, a requirement is specified as a constraint (typically a bound or equality) on the probabilities of one or more events. This includes non-quantitative requirements, which can be viewed as events with probability 1 . Moreover, such probabilities may be conditional, meaning that they are conditioned on the satisfaction of one or more preconditions. For example, the validation effort might initially focus on the survivability of the system, assuming it has been successfully bootstrapped. That would make it possible to deal with the bootstrapping process separately, if needed, perhaps later on in the validation procedure. In the case of $E x R$, we may define:

$E_{E x R}=X$ is delivered successfully upon request.

$C_{E x R}=$ The system has been successfully bootstrapped, which includes starting the application that provides the service $X$.

$E x R=P\left[E_{E x x} \mid C_{E x R}\right] \geq p_{X}$.

Each event (including preconditions) may be stated as a conjunction of simpler events, both for clarity and ease of subsequent requirement decomposition. Since events are technically sets in the context of probability theory, throughout the paper, "conjunction between events" describes the corresponding set intersection.

\section{Requirement Decomposition}

If feasible, it is helpful to logically decompose a requirement into (more specific) sub-requirements, such that if all of the sub-requirements are satisfied by the system, then the original requirement is satisfied. The primary purpose of the decomposition is to obtain sub-requirements that can be proved using the tools available to the validators for logical argumentation and probabilistic modeling. Our formalism is somewhat similar to the formal composition and refinement framework developed by Abadi-Lamport $[16,17]$ and Shankar [18]. However, their logical formulations do not have probabilistic underpinnings capable of dealing with quantitative survivability requirements, such as our formalism aims to provide. More precisely, a logical decomposition (LD) of a requirement $R$ is a set of two or more requirements (the sub-requirements of $R$ ) such that their (logical) conjunction implies $R$. Formally, let $\left\{R_{1}, R_{2}, \ldots, R_{\mathrm{m}}\right\}$ denote the set of subrequirements of requirement $R(m \geq 2)$ and let $\wedge$ denote conjunction (the logic operator AND). Then,

$$
\left(R_{1} \wedge R_{2} \wedge \ldots \wedge R_{\mathrm{m}}\right) \Rightarrow R,
$$


i.e., the conjunction of the sub-requirements (logically) implies $R$. Accordingly, in order to validate the system with respect to $R$, it suffices to validate it with respect to each of the subrequirements. We rule out trivial conjunctions such as $R \wedge R$ and $R \wedge$ Taut, where Taut is a tautology (is always true). We also rule out degenerate LDs for which the conjunction $\left(R_{1} \wedge R_{2} \wedge \ldots \wedge R_{\mathrm{m}}\right)$ is a contradiction (is never true), e.g., the subrequirements are inconsistent, since in that case (by the definition of logical implication), requirement $R$ would be trivially satisfied.

A requirement is decomposable if it admits to an LD. An LD of $R$ is typically determined by finding some initial LD of $R$ and then iteratively determining LDs of decomposable subrequirements. Note that conditions of the LD definition are preserved by such iterations by the transitivity of $\Rightarrow$.

A requirement $R$ is atomic if it is not logically decomposed. This applies to the original requirement (if it is not decomposed) or to any subrequirement that is not further decomposed. It is important to note that the LD process is guided by the need to obtain manageable sub-requirements, so

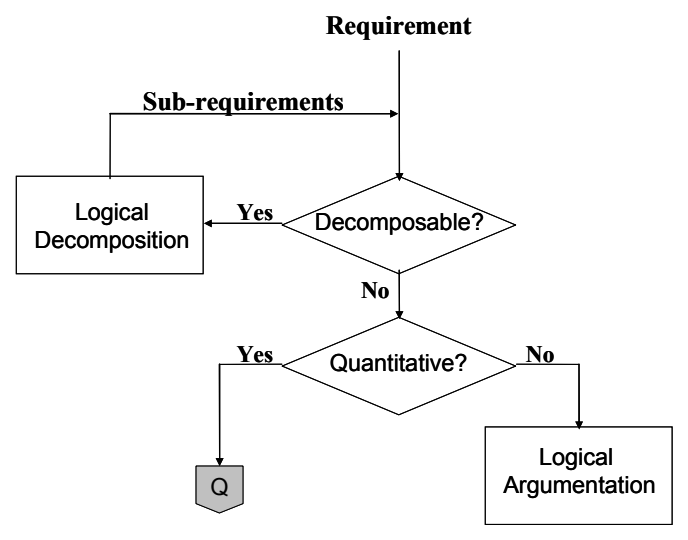

while a requirement may be atomic because it is not decomposable (does not admit to an LD), in practice, a requirement is often atomic because a beneficial LD is not evident or because the requirement is basic enough to be dealt with effectively without further decomposition. If $R$ is decomposed in the iterative manner described above, the decomposition may be visualized as a tree, with $R$ as the root node and the atomic subrequirements as the leaf nodes. It follows (due to the preservation of the LD condition) that $R$ is satisfied if all of its atomic sub-requirements (leaf nodes for the tree rooted at $R$ ) are satisfied.

Generally, the sub-requirements of an LD of $R$ can be either quantitative or non-quantitative (see Section 3.1). If $R$ is quantitative, then at least one of its sub-requirements must also be quantitative. The reason is that logical decomposition cannot eliminate the quantitative aspect(s) of $R$. Moreover, it is possible for an LD of a quantitative requirement to contain more than one quantitative sub-requirement.

Consider the following example. Suppose we have a requirement $R$, defined as

$$
R: P\left[E_{1} \wedge E_{2}\right] \geq p(0<p<1),
$$

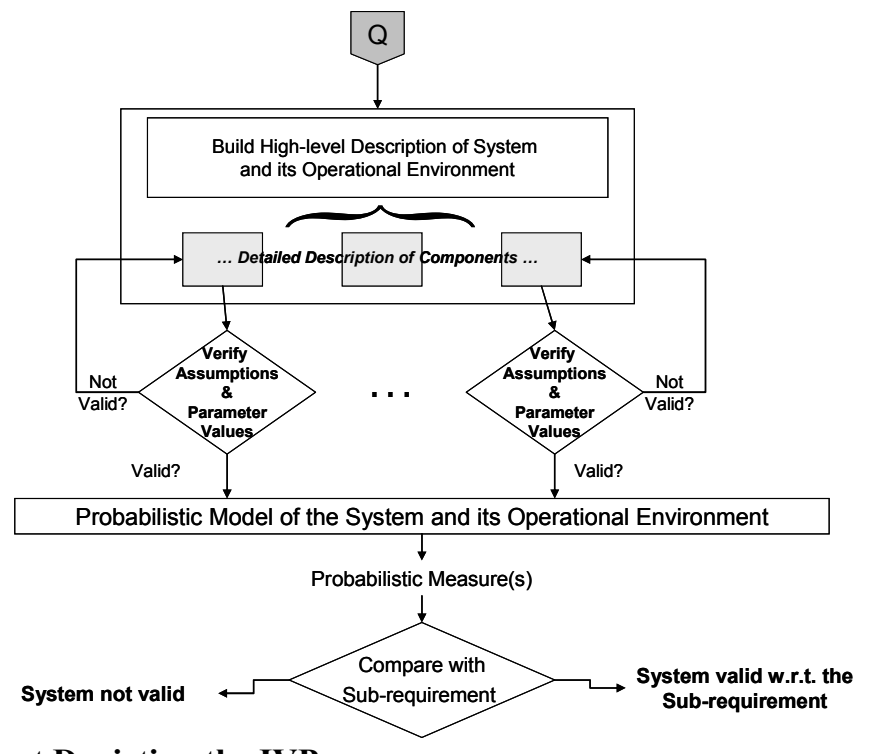

Fig. 4 - A Flowchart Depicting the IVP

where $E_{1}$ and $E_{2}$ are two events, such that neither of them is expected to occur with probability 1. Suppose further that these events are not statistically independent. Nevertheless, $R$ can still be logically decomposed into two sub-requirements

$$
\begin{aligned}
& R_{1}: P\left[E_{1}\right] \geq p_{1}\left(0<p_{1}<1\right), \\
& R_{2}: P\left[E_{2} \mid E_{1}\right] \geq \frac{p}{p_{1}},
\end{aligned}
$$

where it follows from elementary probability theory that $R_{1} \wedge R_{2} \Rightarrow R$. The value of $p_{1}$ can be specified by using a probabilistic model of the relevant portion of the system to evaluate $P\left[E_{1}\right]$ and letting $p_{1}=P\left[E_{1}\right]$, thereby satisfying $R_{1}$. Another model can then be used to evaluate $P\left[E_{2} \mid E_{1}\right]$ so as to determine whether $R_{2}$ is satisfied. Note that (due to the use of implication), it is sufficient to do this for one value of $p_{1}$, and that the independence of the events involved is not a prerequisite for decomposition.

\section{Outline of the Validation Procedure}

Although it is sometimes possible to obtain a validation result by applying a single validation technique (e.g, logical argumentation, if the 
requirement is not quantitative), we find that validation with respect to high-level quantitative survivability requirements calls for an integrated application of several techniques. Indeed, we believe that the means of accomplishing this is a distinguishing feature of the effort reported herein. We used the following integrated validation procedure (IVP) to validate the IT Pub-Sub with respect to a quantitative survivability requirement, say $R$. The quantified aspects of $R$ are assumed to be probabilistic (e.g., probabilities, moments of random variables, and so forth). The IVP is summarized in Fig. 4, and the steps are:

1. Formulate a precise statement of $R$, including any assumed preconditions regarding the system and/or its operational environment. The purpose of this step is to make the goals of the validation exercise absolutely clear right at the onset, and also to specify exactly the scope of the validation by explicitly enumerating the preconditions. We chose propositional logic in combination with a simple probabilistic formulation as the specification formalism, since it easily supports subsequent decomposition as described in Section 3.2.

2. If $R$ admits to logical decomposition, decompose it iteratively using the method described in Section 3.2, thereby determining its corresponding atomic sub-requirements. Otherwise, $R$ is the only atomic requirement. This step is intended to break $R$ into manageable sub-requirements that can be addressed by the tools and techniques used for probabilistic modeling or logical argumentation. Each decomposition of a requirement into subrequirements is accompanied by a proof of the validity of the decomposition. The proofs would typically use propositional logic, and might use some probabilistic reasoning if any of the involved sub-requirements are quantitative.

The following steps are applied to each atomic sub-requirement $R_{\mathrm{a}}$. ( $R_{\mathrm{a}}=R$ if $R$ is atomic.)

3. If $R_{\mathrm{a}}$ is quantitative, proceed as follows; otherwise, jump to Step 8. In a natural language (or some more formal language suited to the task), describe properties of the system and its operational environment that can guide modelbased evaluation of the probabilistic measure(s) associated with $R_{\mathrm{a}}$. First, the system components (and communications among them) relevant to $R_{\mathrm{a}}$ are identified. This is followed by a description of the following. Note that all the descriptions are in terms of the components and communications identified, i.e., they are specified at the same level of abstraction.

a) Information flows: i) Service-related data flows: this is a block diagram, with the components identified above as the blocks, representing the precise sequence, and the nature, of communications between the components during normal operation.

ii) Attack-caused intrusion detection and recovery flows: this is a block diagram representing the precise sequence, and nature, of communications involved in passing alerts (upon detection of intrusions) and subsequent recovery commands.

iii) Fault-caused error detection and recovery flows: similar to (ii) above, except that the alerts are raised upon detection of errors.

b) Use scenario(s): this is closely related to information flows. Here, the operational setting of the system is described, and might include details such as frequencies of various events. A description of the quantitative measure(s) required to evaluate $R_{\mathrm{a}}$ is also provided.

c) Attack and fault effects: This is a description of the possible effects attacks and faults have on the behavior of the system components. The emphasis is on enumerating the effects that can be detrimental to the system's ability to satisfy $R_{\mathrm{a}}$. It usually includes a representation of the attacker behavior, in particular the dynamics of the spread of intrusions deeper into the system using already intruded components as launching pads. These descriptions can be based on information from threat and vulnerability assessment and from whiteboarding.

4. Determine detailed descriptions of submodels corresponding to the relevant components identified in Step 3, along with explicit statements of underlying assumptions made for each submodel. For each submodel, descriptions elaborate on (1) the participation of the component in the various information flows described in $3 \mathrm{a},(2)$ the state to be maintained to evaluate the measure described in $3 b$, and (3) effects of intrusions on the behavior of the component as per 3c. The descriptions are at a level of detail that permits relatively straightforward construction of corresponding portions of the probabilistic model in Step 6. Step 4 also includes identification of the input parameters required by the model, and choice of reasonable estimates for their values.

Steps 3 and 4 help the validators make sure they clearly understand the system design or implementation being validated, and document that understanding. Since the probabilistic model 
constructed in Step 6 may not be easily understood by persons lacking background in probability theory and stochastic modeling, the descriptions prepared in the above steps can be reviewed by the designers/implementors of the system to ensure compliance with their views. Similarly, the descriptions help convince the accreditors that the models actually represent the system being validated. The above exercise also greatly eases the job of building the probabilistic model.

5. Verify the modeling assumptions of Step 4 and, where possible, justify values of the model parameter values chosen. Since we were validating a system design (as opposed to an implementation), several of the parameter values chosen were based on informal justification rather than formal proofs or experimentation. The focus in such a case is more on exploration of the design/parameter space and identification of the subspace that ensures compliance with the survivability requirements, thus leading to a more survivable system when the chosen design is actually implemented. The assumptions are also checked for consistency with any preconditions in $R_{\mathrm{a}}$. Furthermore, if a node in the requirement decomposition tree used independence of the underlying events to justify the decomposition, the sets of assumptions in the subtrees rooted at the children of that node are checked against each other for the violation of the independence assumption.

6. Based on the descriptions obtained in Step 4, construct a probabilistic model of the system and its operational environment that can support evaluation of the probability measure(s) associated with $R_{\mathrm{a}}$. Several modeling formalisms may be used. We have used Stochastic Activity Networks (SANs) [19], a generalization of stochastic Petri nets, as the modeling formalism. The models were built using the Möbius tool, which can either solve them analytically by converting them into equivalent continuous time Markov chains, or simulate them by executing multiple behavioral trajectories until the measures being evaluated are determined within desired bounds of accuracy.

7. Based on the model made in Step 6, evaluate the probability measure(s) associated with $R_{\mathrm{a}}$. If the values obtained are within bounds prescribed by $R_{\mathrm{a}}$, then $R_{\mathrm{a}}$ is satisfied by the system (the system is valid with respect to $R_{\mathrm{a}}$ ).

8. If $R_{\mathrm{a}}$ is not quantitative, prove that it is satisfied using logical argumentation.

Note that Steps 4-5 will usually be iterated. For example, an inability to verify some assumption in
Step 5 may lead to alternative assumption details in Step 4 (even though realities of the design and its operational environment remain unchanged).

\section{VALIDATION DETAILS}

As mentioned in Section 2, the essential services provided by the core to clients are publish, subscribe, and query. Accordingly, IT Pub-Sub survivability with respect to these services is a dominant concern of the validation process. We now describe the application of the validation procedure outlined above as it was used to validate the system against the survivability requirement for the publish service.

\section{Step 1: Requirement Specification}

The "capability to process a publish request successfully" was chosen as the survivability requirement for the publish service. The first step in the validation procedure is to formulate a precise statement of the requirement. To this end, the terms "capability" and "successfully process a publish request" need to be defined, together with any preconditions regarding the system and its operational environment.

Let $C_{P U B}$ be the conjunction of the events representing the preconditions. In our case, $C_{P U B}$ is the conjunction of the following two events; the first refers to the system and the second to the use environment.

$C_{P U B}^{1}=$ the publishing client is successfully registered with the IT Pub-Sub core (authentication).

$C_{P U B}^{2}=$ the publishing client's mission application always passes adequate and accurate information to the client.

These reflect the assumptions about the system's initial state and invariants during operation, which are taken as axioms in the subsequent analysis.

Let $E_{P U B}$ be the desired event, i.e., the successful processing of a request to publish. It is the conjunction of the following events.

$E_{P U B}^{1}=$ the data flow of the publish operation is correct.

$E_{P U B}^{2}=$ the time required for the publish operation does not exceed a specified duration $t_{\max }$ (timeliness).

$E_{P U B}^{3}=$ the published IO that becomes available to subscribers has the same essential content as that assembled by the publishing client (integrity).

$E_{P U B}^{4}=$ the published IO is available only to the other clients via subscribe or query requests (confidentiality).

Let $P\left[E_{P U B} \mid C_{P U B}\right]$ be the probability of event $E_{P U B}$, given that the preconditions hold, i.e., the 
quantification of "capability." Let the required capability be denoted by $p_{P U B}\left(0<p_{P U B}<1\right)$, the lower bound on $P\left[E_{P U B} \mid C_{P U B}\right]$, where its specified value is typically a high probability that depends on the nature of the system use scenario.

The survivability requirement for the IT PubSub publish service, denoted by PUB, can then be stated as

$$
\text { PUB: } P\left[E_{P U B} \mid C_{P U B}\right] \geq p_{P U B} \text {. }
$$

PUB is therefore a quantitative requirement (in the sense described in Section 3.1), since deciding whether it is satisfied by the IT Pub-Sub, entails evaluation of the quantitative measure $P\left[E_{P U B} \mid C_{P U B}\right]$. Due to uncertainties in attacks, attack effects (intrusions), intrusion effects, and the operation of various IT Pub-Sub intrusion tolerance mechanisms, validation is trivial in the case $p_{P U B}=$ 1 , since we know that PUB cannot be satisfied. At the other extreme, if the value $p_{P U B}=0$ were allowed and so specified, then PUB would likewise be non-quantitative. Again, validation would be trivial, since, in this case, PUB would always be satisfied (the requirement itself is trivial).

\section{Step 2: Logical Decomposition}

PUB can be initially decomposed into the following two sub-requirements.

$$
\begin{aligned}
& \mathrm{PUB}_{1}: P\left[E_{P U B} \mid C_{P U B}^{2}\right] \geq p_{P U B} . \\
& \mathrm{PUB}_{2}: P\left[C_{P U B}^{1}\right]=1 .
\end{aligned}
$$

To establish that $\left\{\mathrm{PUB}_{1}, \mathrm{PUB}_{2}\right\}$ is an $\mathrm{LD}$ of PUB, we need to show that the defining conditions of an LD are satisfied, i.e.,

$$
\left(\mathrm{PUB}_{1} \wedge \mathrm{PUB}_{2}\right) \Rightarrow \mathrm{PUB} \text {. }
$$

Suppose the hypothesis holds, i.e., both $\mathrm{PUB}_{1}$ and $\mathrm{PUB}_{2}$ are true. Generally, if $A$ and $B$ are two events such that $P[A]=1$ then

$$
\begin{aligned}
P[A \wedge B] & =P[A]+P[B]-P[A \vee B] \\
& =P[B]=P[A] P[B]
\end{aligned}
$$

where $P[A \vee B]=1$, since the probability of an "or" event is at least the probability of either disjunct, and can be no greater than 1. From the above identity, it follows that any event having probability 1 is (statistically) independent of an arbitrary event. In particular, by $\mathrm{PUB}_{2}, C_{P U B}^{1}$ is independent of both $E_{P U B}$ and $C_{P U B}^{2}$. Hence,

$$
\begin{aligned}
P\left[E_{P U B} \mid C_{P U B}^{2}\right] & =\frac{P\left[E_{P U B} \wedge C_{P U B}^{2}\right]}{P\left[C_{P U B}^{2}\right]}=\frac{P\left[E_{P U B} \wedge C_{P U B}^{2}\right] P\left[C_{P U B}^{1}\right]}{P\left[C_{P U B}^{2}\right] P\left[C_{P U B}^{1}\right]} \\
& =\frac{P\left[E_{P U B} \wedge C_{P U B}^{1} \wedge C_{P U B}^{2}\right]}{P\left[C_{P U B}^{1} \wedge C_{P U B}^{2}\right]}=P\left[E_{P U B} \mid C_{P U B}^{1} \wedge C_{P U B}^{2}\right] \\
& =P\left[E_{P U B} \mid C_{P U B}\right]
\end{aligned}
$$

Since $P\left[E_{P U B} \mid C_{P U B}^{2}\right] \geq p_{P U B}$ (sub-requirement $\left.\mathrm{PUB}_{1}\right)$, by the above identity we can conclude that

$$
P\left[E_{P U B} \mid C_{P U B}\right] \geq p_{P U B},
$$

which is just the original requirement PUB. Hence, the LD condition holds.

Regarding the four events that define $E_{P U B}$, a study of the design reveals that integrity $\left(E_{P U B}^{3}\right)$ and confidentiality $\left(E_{P U B}^{4}\right)$ can likewise be regarded as probability-1 events, given that precondition $C_{P U B}^{2}$ is satisfied (event $C_{P U B}^{2}$ occurs). More precisely, we have the following subrequirements of requirement $\mathrm{PUB}_{1}$.

$$
\begin{aligned}
& \mathrm{PUB}_{1,1}: P\left[E_{P U B}^{1} \wedge E_{P U B}^{2} \mid E_{P U B}^{3} \wedge E_{P U B}^{4} \wedge C_{P U B}^{2}\right] \geq p_{P U B} \\
& \operatorname{PUB}_{1,2}: P\left[E_{P U B}^{3} \mid C_{P U B}^{2}\right]=1 \\
& \operatorname{PUB}_{1,3}^{2}: P\left[E_{P U B}^{4} \mid C_{P U B}^{2}\right]=1
\end{aligned}
$$

To insure that $\left\{\mathrm{PUB}_{1,1}, \mathrm{PUB}_{1,2}, \mathrm{PUB}_{1,3}\right\}$ is an $\mathrm{LD}$ of $\mathrm{PUB}_{1}$, we must again show that the constraint for LD holds, i.e.,

$$
\left(\mathrm{PUB}_{1,1} \wedge \mathrm{PUB}_{1,2} \wedge \mathrm{PUB}_{1,3}\right) \Rightarrow \mathrm{PUB}_{1} .
$$

Suppose the above hypothesis holds $\left(\mathrm{PUB}_{1,1}\right.$, $\mathrm{PUB}_{1,2}$, and $\mathrm{PUB}_{1,3}$ are true). Extending observation (1) to conditional probabilities, it follows from $\mathrm{PUB}_{1,2}$ and $\mathrm{PUB}_{1,3}$ that $P\left[E_{P U B}^{3} \wedge E_{P U B}^{4} \mid C_{P U B}^{2}\right]=1$. Using this fact together with the sub-requirements $\mathrm{PUB}_{1,2}$ and $\mathrm{PUB}_{1,3}$,

$$
\begin{aligned}
P\left[E_{P U B}^{1} \wedge\right. & \left.E_{P U B}^{2} \mid E_{P U B}^{3} \wedge E_{P U B}^{4} \wedge C_{P U B}^{2}\right] \\
& =\frac{P\left[E_{P U B}^{1} \wedge E_{P U B}^{2} \wedge E_{P U B}^{3} \wedge E_{P U B}^{4} \wedge C_{P U B}^{2}\right]}{P\left[E_{P U B}^{3} \wedge E_{P U B}^{4} \wedge C_{P U B}^{2}\right]} \\
& =\frac{P\left[\left(E_{P U B}^{1} \wedge E_{P U B}^{2} \wedge E_{P U B}^{3} \wedge E_{P U B}^{4}\right) \wedge C_{P U B}^{2}\right]}{P\left[E_{P U B}^{3} \wedge E_{P U B}^{4} \mid C_{P U B}^{2}\right] P\left[C_{P U B}^{2}\right]} \\
& =\frac{P\left[E_{P U B} \wedge C_{P U B}^{2}\right]}{1 \cdot P\left[C_{P U B}^{2}\right]}=P\left[E_{P U B} \mid C_{P U B}^{2}\right]
\end{aligned}
$$

Since $P\left[E_{P U B}^{1} \wedge E_{P U B}^{2} \mid E_{P U B}^{3} \wedge E_{P U B}^{4} \wedge C_{P U B}^{2}\right] \geq p_{P U B}$ by $\mathrm{PUB}_{1,1}$, we conclude from the above identity that $\mathrm{PUB}_{1}$ is true.

Each sub-requirement available at this stage is specific enough to be handled by a validation technique such as probabilistic modeling or logical argumentation. Hence, we stop the decomposition, and refer to $\mathrm{PUB}_{1,1}, \mathrm{PUB}_{1,2}, \mathrm{PUB}_{1,3}$, and $\mathrm{PUB}_{2}$ as the atomic sub-requirements of PUB, where the first is quantitative and the other three are nonquantitative.

\section{Step 3: High-Level System Description}

For the remaining steps of the validation, we will focus on the quantitative atomic requirement $\mathrm{PUB}_{1,1}$. With respect to $\mathrm{PUB}_{1,1}$, this step seeks high-level descriptions of the IT Pub-Sub data flow 
for the publish operation, the associated alert and response data flows, and the attack/intrusion environment. The descriptions guide subsequent determination of the detailed descriptions needed to construct a probabilistic model that supports evaluation of the probability measure $P\left[E_{P U B}^{1} \wedge E_{P U B}^{2} \mid E_{P U B}^{3} \wedge E_{P U B}^{4} \wedge C_{P U B}^{2}\right]$ associated with $\mathrm{PUB}_{1,1}$.

\subsubsection{Data Flows}

Both the publish data flow and the attack/alert data flow are represented as block diagrams depicting both the relevant components and the sequence of operations and nature of the communication between the components. The diagrams (Fig. 2 and Fig. 3) and the associated descriptions have already been provided as a part of Section 2 and are not repeated here.

\subsubsection{Attack Model}

The attack model makes several important distinctions concerning where attacks occur (location of both the source and target of an attack) and how resulting intrusions affect both system and attacker behavior. In particular, the model accounts for the fact that once a vulnerability has been discovered in a target, the attack can quickly propagate to other instances of that target, provided that they are accessible (via network connectivity) from the attack source. If an intruded target (e.g., a host) is compromised, then it is possible for the host to serve as a source of further attacks.

Terminology In order to describe the attack model more precisely, we make the following distinctions. An entity of the system is one of the following:

- A host: A computing resource with an operating system and network interface cards.

- A component: A process that realizes an IT PubSub function, e.g., an access proxy. Note that several components typically reside in a single host (for example, the survivability delegate, the sensors, the actuator, and the local controller reside in the client).

- A process domain (PD): An entity that implements a component, e.g., the sensor process domain implements the sensor component, and the AP IO (AP_IO) process domain implements the AP that deals with forwarding IOs.

- An application: The application level of the process domain.

An intrusion is a possible outcome of an attack. It occurs if the attack finds a vulnerability in its target and thereby alters the target's behavior (the effect or symptom of the intrusion); otherwise, it is prevented. In other words, an intrusion occurs if an attack has some effect on the target. The effect can range from something very benign (e.g., when the intrusion is "masked" or "blocked") to the compromise of the target such that it can be used as a platform for launching further attacks. An intrusion is prevented if the attack can find no vulnerabilities in its target, thereby obviating any effect. In particular, if an attack is unable to access its target, then the attack cannot find a vulnerability, even if one exists.

An intrusion is tolerated (possibly in the presence of other tolerated intrusions) if its effect does not lead to unsuccessful processing of a publish request; otherwise, it causes a failure.

A new vulnerability, found at time $t$, is a vulnerability that is present in at least one component of the architecture, and that was not known by the attacker until time $t$ during the mission. When discovered, such a vulnerability can be used any number of times (until the end of the mission) against the vulnerable components that the attacker can reach.

A successful attack is repeated if that attack (same source, same type) is made on a similar target having the same vulnerability, in which case it succeeds very quickly.

A successful attack is propagated if the intruded target is compromised such that the target becomes a source of further attacks. If this source can access a similar target with the same vulnerability, the original attack can be repeated (see above). The source can also launch a new attack that attempts to find a new vulnerability in another target.

The simulation model considers attacks that are "successful" in the sense that an intrusion occurs and, moreover, is neither masked nor blocked. However, if such an intrusion is tolerated (the third case noted above), the attack does not succeed in the more usual sense of causing a failure.

Attack Propagation Two basic assumptions underlie the construction of the attack model. First, we assumed that the attacker would discover new vulnerabilities slowly. Define MTTD to be the mean time to discovery of a new vulnerability. Second, it was assumed that the attacker would exploit newly discovered vulnerabilities quickly. Once an entity is intruded following the discovery of a vulnerability, the attack can be repeated (see the preceding terminology). Define MTTE to be the mean time between successive exploitations of a known vulnerability. The typical value of MTTE in our analysis was 5 minutes. It is important to note, however, that repeated attacks require targets with the same vulnerability, typically entities that are instances of the original target. Accordingly, design diversity can be used to reduce the possibility of 
repeated attacks. For example, a successful OSlevel attack from the outside, compromising a client running under OS1, can propagate to hosts connected to the client (namely, the access proxy in the core). If the access proxy's OS is also OS1, the attack can be repeated, with success (intrusion) coming quickly. On the other hand, if the access proxy is running under a different operating system, then the OS diversity will likely preclude a repeated attack. It is possible that a given vulnerability exists in more than one OS. We account for that possibility using a probability of a common-mode vulnerability. If a vulnerability is determined to be common-mode, it will exist in all OSes used by the IT Pub-Sub.

Types of Attacks Three different types of attacks were represented in the attack model.

- Infrastructure-level attacks exploit a vulnerability found either in the operating system running on a given host (for instance, a flaw in the TCP/IP stack), or in a service running on that host, not related to the IT PubSub (for example, a flaw in sshd). The attack's source and target must be directly connected and communicate with each other, typically with standard network protocols. If a vulnerability of that type is discovered, it can be limited to one of the four operating systems, or affect all operating systems used in the system.

- Data-level attacks exploit a vulnerability in an IT Pub-Sub application on the targeted component. The attack's source and target are applications on different hosts. The attack uses the content of the application data to intrude into the target. For example, the client sends a corrupted IO to a PSQ server, resulting in a crash or a corruption of that host.

- Attacks across process domains allow the attacker to intrude into a different process domain of the same host by exploiting low-level vulnerabilities in the operating system. The source and targets are process domains running on the same machine.

Successful infrastructure-level attacks are attacks in depth; the intruder quickly progresses deeply into one quadrant, because only one vulnerability needs to be discovered to compromise the common operating system in the entire quadrant. However, unless the vulnerability found corresponds to a common mode failure, the attacker cannot intrude into any other quadrant, because the other quadrants are based on hosts with different operating systems. Data-level attacks provide attacks in breadth; if a vulnerability is found in the access proxy application, then the publishing client can exploit it directly on the four access proxies.
Those attacks are consequently much more dangerous. Great effort should be put into reducing the number of data vulnerabilities, and preventing attackers from exploiting them.

The first attack on the IT Pub-Sub must be an infra-structure-level attack, since both of the other types require control of a machine within the system. For example, attacks across process domains assume a compromised process domain for the source of the attack. Data-level attacks require control of a component that is capable of generating IT Pub-Sub protocol packets, signed with signatures from host-specific private keys.

Fig. 5 provides an example of attack propagation. At time $t_{0}=85$ minutes, an infrastructure-level vulnerability (ILV) is found on the main process domain of OS1. After a short time, the attacker exploits that vulnerability on the publishing client (time $t_{1}$ ). From the client, he launches the same attack on the AP of quadrant 1 at time $t_{2}$. He continues in the same way until he has compromised all the components of quadrant 1 (since they're all running the same OS). At time $t_{8}$ $=230$ minutes, a data-level vulnerability (DLV) on the PSQ server is found. The attacker uses that vulnerability to compromise the three remaining PSQ servers; he can attack either from the publishing client (times $t_{9}$ and $t_{11}$ ), or from the PSQ server in the first quadrant (time $t_{10}$ ), since he has control of all those entities.

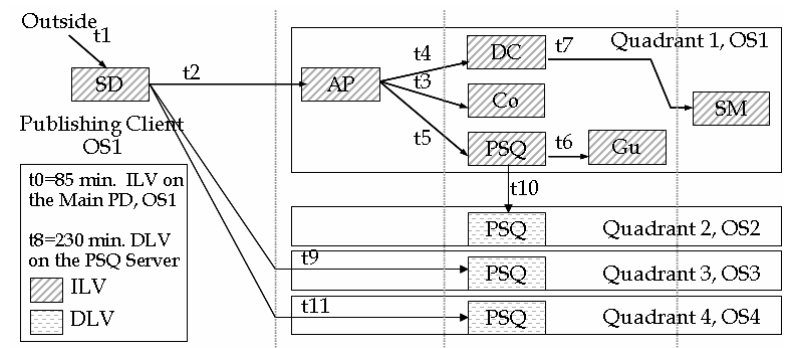

Fig. 5 - Example of Attack Propagation

Intrusion Effects When any of the three types of attacks described above is successfully exploited, the resulting intrusion can have one of the following effects.

- Crash of the intruded entity: For the operating systems that handle process domains (secure $O S e s$ ), a crash of a given process domain will not lead to the crash of other process domains. On a traditional $O S$ (without process domains), a crash of a given process will crash the whole machine (and thus all the processes running on it).

- Compromise of the intruded entity: The intruded entity can corrupt or drop data packets, or can be a source of further attacks. When a process 
domain becomes compromised on a secure operating system, the other process domains are unaffected. However, for traditional operating systems, compromise of one process also compromises the other processes on the machine.

- Denial of service: The intruded entity can reduce processing speeds and increase latencies.

Attack Responses The model includes three mechanisms for responding to a detected intrusion that is not blocked:

- Type 1: Rapid response. The first and fastest mechanism is a local and rapid response, based on an automatic decision made by the LC. Examples of rapid response include file restoration, termination of illegal processes, and restarting of critical processes. On average, this operation takes one minute.

- Type 2: Secure reboot. This operation is carried out in two phases: automatically shutting down the host and then manually restarting it. The shutdown is performed by the SM through DC and LC. Restarting has to be done manually by the operator sitting at the console. The reason is that the client application will also need to be restarted, which will require the user to perform some action, such as providing a password or swiping a smart card. Moreover, the shutdown will give the client operator the opportunity to introduce diversity, perhaps by removing some services or changing the firewall policies.

- Type 3: Permanent isolation. The decision to isolate is taken by the SM through the PS. The decision is made by the SM in the core; therefore, this operation requires more time, on average 7 minutes.

\section{Step 4: Detailed Descriptions}

This step elaborates the Step 3 descriptions to an extent that permits relatively straightforward construction of corresponding components of the probabilistic model. It also explicitly enumerates all the assumptions the probabilistic model makes that are verified, and, if found to be correct, justified, using logical arguments. For the purpose of illustration, we will limit our attention to the AP component, along with certain details concerning the use and attack models.

If the AP is not compromised, it forwards designated traffic from the Quadrant Isolation Switch to core quadrant components and vice versa. The AP, like several other components in IT PubSub, has process domains, which are similar to sandboxed virtual machines running on the same host. The AP handles different types of traffic, and each type, including IOs sent from publishing clients to the core, is handled by a different process domain. If an IO is sent by a publishing client to the AP (the client sends the IO to a randomly chosen quadrant), the token accompanying the IO is sent to the quadrant's DC to assist in the determination of whether the publishing client is in a session. If the DC's response is positive, the IO is forwarded to the PSQ server.

In addition, the AP forwards sensor alerts from client-side sensors to the correlator, heartbeats from client components to the DC, acknowledgments from PSQ servers to clients, notifications from PSQ servers to subscribing clients, commands from the DC to clients' LCs, and commands from ADF policy servers to clients' ADF NICs.

The following assumptions are identified in the model for the AP component:

AP1: Only well-formed traffic is forwarded by a correct AP.

AP2: An AP can change the traffic through it, but cannot re-sign the content.

AP3: An AP cannot access the contents of an IO if application-level end-to-end encryption is being used.

AP4: If the AP is compromised, it can launch ILA to the following components: client, PSQ, DC, correlator, and PS.

AP5: If the AP is compromised, it can launch DLA to the following components: PSQ, guardian, DC, and the publish-subscribe middleware components, as well as the IDS components on the clients, correlator, and SM.

The time-to-discovery (TTD) and time-to-exploit (TTE) are assumed to be exponentially distributed. Accordingly, the values assigned to MTTD and MTTE (see the description of the attack model above for definition) parameters of the attack model will be such that MTTE «MTTD. This reflects the fact that the TTD of a new vulnerability is typically much longer than its subsequent TTE.

Once an intrusion has occurred, it has one of the three possible effects described in Step 3 (crash, compromise, or DoS), which we assume will occur with probabilities (parameters of the attack model) $p_{\text {cr, }}, p_{\text {co }}$, and $p_{\text {dos, }}$ respectively. When a process domain on an AP is compromised, data that flows through it can be altered as follows. If any alert and command traffic passes through, it is dropped (blocked). If the process domain handles IOs, it will corrupt an IO if it has access to the client's key (since the process domains can change the signature of the IO according to the corrupted content); otherwise, it will drop the IO. It can also act as a source for further attacks (both ILA and DLA) on other entities. Compromised sensors are not able to detect intrusions. When an alert is generated by the IDS, the recovery options are as follows. On the 
first alert, the affected process domain is restarted. On the next three, the entire AP is rebooted. On the fifth alert, the AP is quarantined (using the ADF NICs).

\section{Step 5: Justification of the Assumptions}

The goal in this step is to justify the model assumptions made in Step 4. We used logical argumentation in this step. Other techniques, such as formal methods and experimentation, were also utilized for some of the assumptions. In the complete description of the model in Step 4, a total of 31 assumptions were justified using logical argument and experimental results. These arguments use very low-level system details, and, in the interest of space, we do not list them here. Interested readers can refer to [20] for details.

\section{Step 6: Construction of the Probabilistic Model}

Based on detailed descriptions of the type illustrated in Step 4, a probabilistic model was constructed using Möbius [21] that supports evaluation of the probability measure $P\left[E_{P U B}^{1} \wedge\right.$ $\left.E_{P U B}^{2} \mid E_{P U B}^{3} \wedge E_{P U B}^{4} \wedge C_{P U B}^{2}\right]$. We used stochastic activity networks (SANs) [19] as the formalism. Behaviorally, a model constructed with SANs represents (measure-relevant) behavior of the IT Pub-Sub platform in the presence of publish demands and random attack-caused intrusions. Structurally, the atomic SAN submodels represent various components of the design and its use/attack environment; descriptions of them were documented during Step 4. The overall model is constructed by using replicate and join operations to compose the atomic submodels.

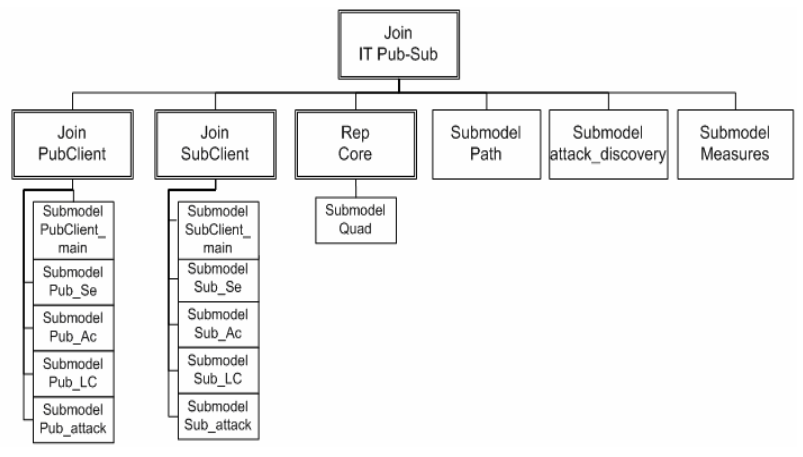

Fig. 6 - Composed Model of the IT Pub-Sub

Graphically, a composed model can be viewed as a tree, in which the atomic SAN submodels correspond to leaf vertices of the tree, and joins or replicates correspond to internal vertices. A Join vertex combines two or more different SAN submodels, each of which can itself be a composed model (represented by a subtree). A Rep (or
Replicate) node generates multiple copies of its submodel (again, each submodel can itself be a composed model). Different submodels in a composed SAN interact through shared state variables.

As depicted in Fig. 6, the system is viewed (for the purpose of validating the requirement $\mathrm{PUB}_{1,1}$ ) as two clients (Join PubClient and SubClient) communicating with the core (Rep Core) through the network (submodel Path). The clients have four process domains, represented by four submodels under the Join (IT Pub-Sub publish functionality, the sensors, the actuator, and the local controller). The fifth submodel under each of these Joins is the attack model. The two remaining submodels (measures and attack_discovery) assist modelbased formulation of the measures evaluated.

The core is a replication of four quadrants (submodel Quad). As shown in Fig. 7, each quadrant is a Join of several submodels (Access Proxy, PSQ, DC, Guardian, Correlator, PS, and $S M)$, representing all of the core components. As presented in Fig. 1, the access proxy, PSQ server, downstream controller, and guardian have IDS components; therefore, their respective Joins have IDS submodels (for instance, $A P_{-} S e, A P_{-} A c$, and $\left.A P_{-} L C\right)$.

For each node represented in the composed models of Fig. 6 and Fig. 7, an atomic SAN model is built. Atomic SANs encode the state variables representing the components they are modeling, and provide transitions with specified delay distributions that can change the state. They provide the ability to include complex enabling functions for the transitions, and complex completion functions to manipulate the state upon completion of transitions. Each atomic SAN basically implements the description for the corresponding component in Step 4. A detailed description of all the atomic SANs constructed is provided in [22].

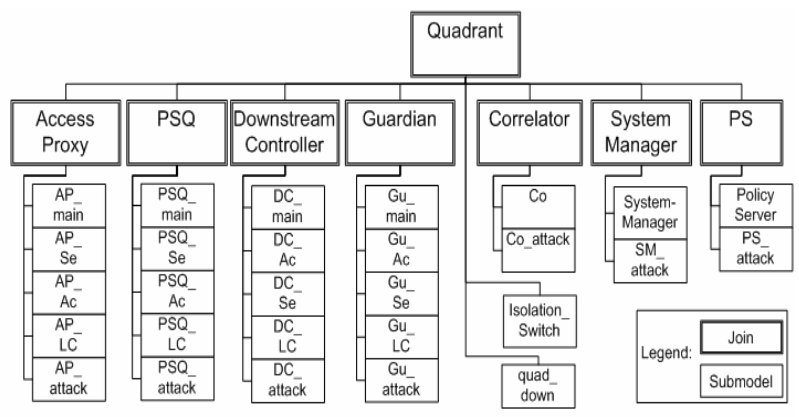

Fig. 7 - Composed Model of the IT Pub-Sub Core Quadrant

The point to note is that the entire model construction is driven by the measure we intend to evaluate. In particular, the SANs have state variables maintaining a count of the number of 
publish attempts by the clients, and the number of those attempts for which the events $E_{P U B}^{1} \wedge E_{P U B}^{2}$ occur. Furthermore, the construction of the model always assumes $E_{P U B}^{3}, E_{P U B}^{4}$, and $C_{P U B}$ are given, and thus does not include any state for keeping track of those events.

\section{Step 7: Evaluation for Validation Against Requirement}

Based on the model of step 6 , this step evaluates the probability measure

$$
p_{1,1}=P\left[E_{P U B}^{1} \wedge E_{P U B}^{2} \mid E_{P U B}^{3} \wedge E_{P U B}^{4} \wedge C_{P U B}^{2}\right]
$$

associated with the atomic quantitative requirement $\mathrm{PUB}_{1,1}$. If $p_{1,1} \geq p_{P U B}$ (the required lower bound),

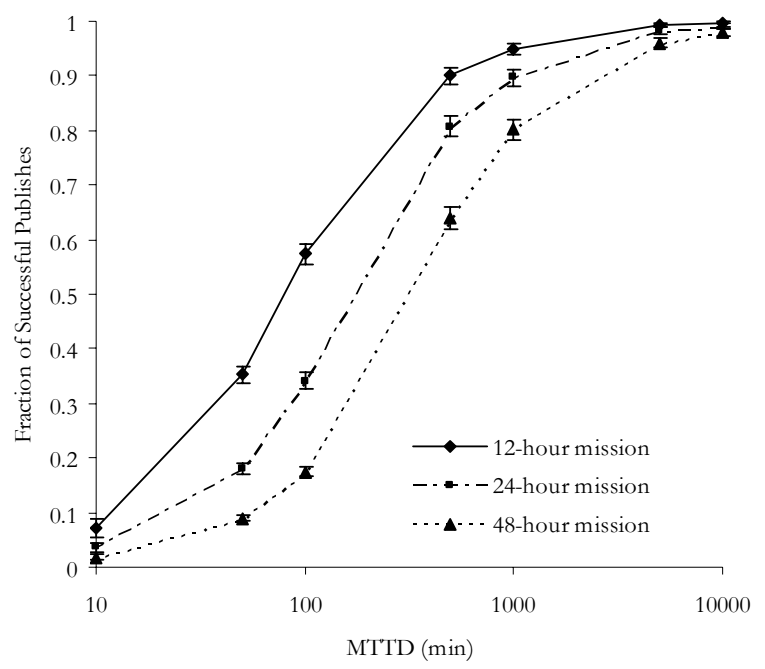

(a) $p_{1,1}$ versus $M T T D$ (minutes) then $\mathrm{PUB}_{1,1}$ is satisfied by the system (the system is valid with respect to $\mathrm{PUB}_{1,1}$ ).

The IT Pub-Sub system is envisaged to be used for short (of the order of a day or two) durations. Letting $d_{\mathrm{M}}$ denote the duration (in hours) of a use scenario, $p_{1,1}$ is formulated as the fraction of publication requests during $d_{\mathrm{M}}$ that are processed successfully, i.e., the fraction for which the event $E_{P U B}^{1} \wedge E_{P U B}^{2}$, given $E_{P U B}^{3}, \quad E_{P U B}^{4}$, and $C_{P U B}^{2}$, occurs. For three different choices of $d_{\mathrm{M}}$, Fig. 8(a) displays the values of $p_{1,1}$ as a function of the MTTD of a new vulnerability (in minutes). Those vulnerabilities are assumed to be exploited very quickly, i.e., $M T T E=2$ minutes. The publishing client publishes a new IO every 5 minutes.

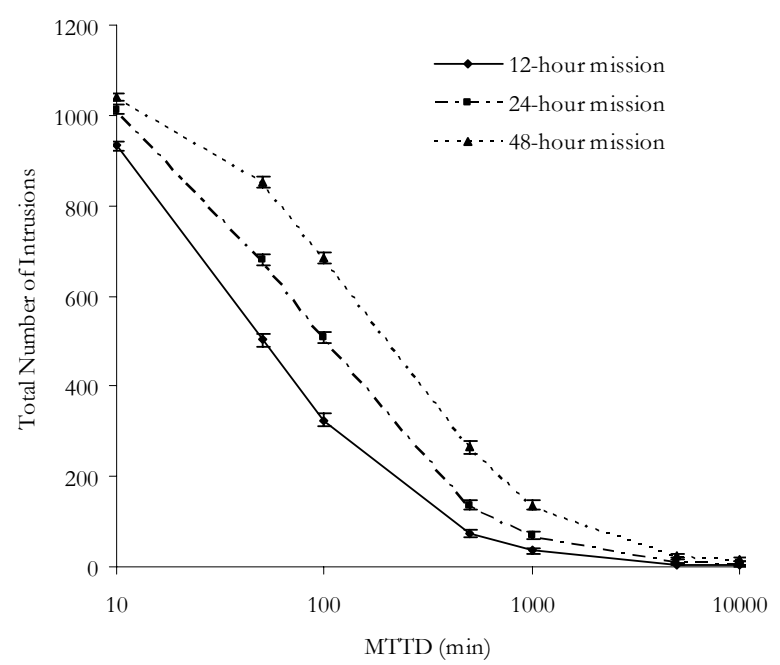

(b) Total number of intrusions versus MTTD (minutes)

Fig. 8 - Variation of Measures with Change in MTTD

In particular, if (1) new vulnerabilities are discovered at an average rate of no more than once per day $(M T T D \geq 1440$ minutes), which is a fairly aggressive assumption, (2) the value of $p_{P U B}$ used is 0.95 , and (3) the mission duration is 12 hours, then the IT Pub-Sub is valid with respect to $\mathrm{PUB}_{1,1}$.

In addition to measures associated directly with quantitative survivability requirements, the model constructed in Step 5 is sufficiently detailed to support evaluation of a variety of other survivabilityrelated measures. For example, to understand how the number of successful attacks (those that cause intrusions) relates to a value of $p_{1,1}$, the measure

$$
n=\text { the total number of intrusions during } \mathrm{d}_{\mathrm{M}}
$$

can likewise be evaluated as a function of MTTD. Comparing Fig. 8(a) with Fig. 8(b), we see how the probability $p_{1,1}$ of successfully processing a publish request during $d_{\mathrm{M}}$ varies inversely with the total number $n$ of successful intrusions experienced during a mission of the same duration.

\section{Step 8: Satisfaction of Non-quantitative Atomic Requirements}

Validation with respect to the non-quantitative atomic requirements $\mathrm{PUB}_{1,2}, \mathrm{PUB}_{1,3}$, and $\mathrm{PUB}_{2}$ is accomplished using logical argumentation. Given that validity has been established with regard to the quantitative atomic requirement $\mathrm{PUB}_{1,1}$ in Step 7 above, the system is then valid with respect to the original requirement PUB. The argumentation used is similar to that used for validation of assumptions, and in the interest of space we do not provide the details here. Details are presented in [20].

\section{Other Evaluation Results: Exploring Design Choices}

In addition to validating the system against the high-level survivability requirements, we also used the probabilistic model developed as a part of the IVP application to study design trade-offs and explore the behavior of the system in different operating configurations and attack environments. 
Such studies are possible because each component of the system model contains a set of input parameters that define aspects of the component's functionality, such as the rate of data publishes or the type of operating system used on a host. These are in addition to parameters that define the characteristics of the attackers on the system, such as the time between vulnerability discoveries (MTTD) and the time to exploit known vulnerabilities (MTTE).

We present results for two of the studies performed: effect of the degree of operating system diversity among the four quadrants in the core and effect of the autonomous distributed firewall (ADF NIC) policies.

\subsubsection{Effect of Diversity}

In this experiment, we studied two different OS configurations in the following way:

- 4 OS: the access proxy, downstream controller, PSQ servers, and system manager in a given quadrant run the same operating system. Each quadrant uses a different OS. The policy server is available in only one operating system. Therefore, there is no possible variation for this component. This case corresponds to the default configuration of the system.

- $1 O S$ : all the components in the core (apart from the policy servers) run the same operating system.

Also, to make a fair comparison between the two options, all OSes are secure.

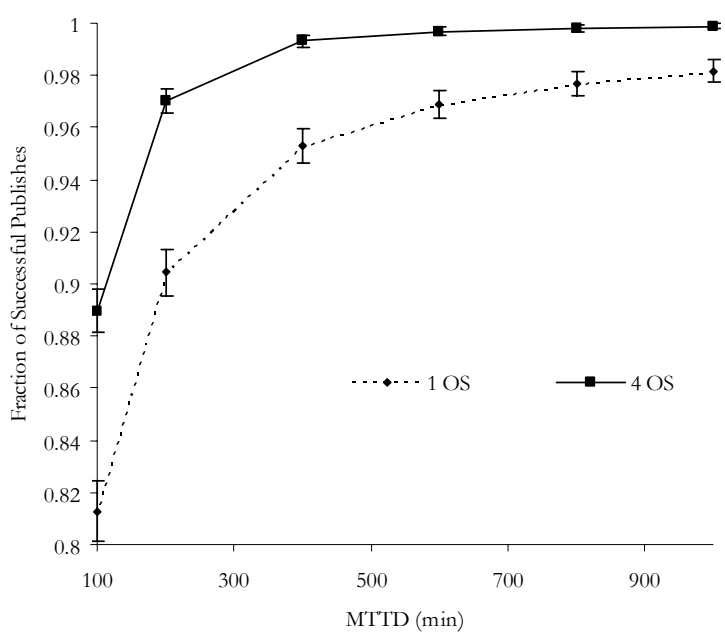

Fig. 9 - $p_{1,1}$ versus MTTD: 4 OS vs 1 OS

The graph presented in Fig. 9 is based on an assumption of a less aggressive attacker than we considered in the baseline case; here, the attacker can only execute infrastructure attacks and attacks across process domains. The figure illustrates the difference between the two options mentioned above. It shows $p_{1,1}$ versus the vulnerability discovery rate. Diversity significantly increases the performance of the design: for $M T T D=200$ minutes, $p_{1,1}$ is about 0.97 for the 4-OS case, versus 0.90 for 1 OS, i.e., a $70 \%$ improvement of the unavailability $(0.10$ versus 0.03$)$. The gap between the two curves is noticeable at all rates.

A similar experiment was done for all types of attack, including the data-level attacks. In that case, the two curves were closer. Data-level attacks are the most dangerous type of attack, as they can take out the same component in every quad. For example, a compromised client could launch a data-level attack against the four PSQ servers, which could result in the crash (or compromise) of all four. If that happened, no further PSQ requests would be handled, and the core would be considered down. For the results presented here, we assumed that the data-level vulnerabilities could be considerably reduced not only by the effort put into the implementation of, for example, the PSQ, but also by the semantic checks done on the access proxy for any incoming traffic to the core.

\subsubsection{ADF NIC Policies}

ADF NICs are local firewalls on each component, administered by the policy servers in the core. The third experiment compares three ADF NIC policies, assuming that only infrastructure-level attacks and attacks across process domains are allowed. The first policy is to allow all communications between any two processes of any two components. The second is a per-component policy, allowing only certain components to communicate with each other (for instance, the AP can talk to the PSQ server in its quad, but the client cannot communicate directly with any PSQ server). Finally, the third one is a per-process-domain policy, restricting communications between specific processes.

Fig. 10 presents the results reflecting the three configurations. The graphs reveal that the perprocess-domain policy is by far the best of all three: when $M T T D=100$ minutes, $p_{1,1}=84.4 \%$ for the norestriction policy, versus $90.0 \%$ for the percomponent, and $98.5 \%$ for the per-process-domain one, which corresponds to a $90 \%$ improvement of the unavailability (from $15.6 \%$ down to $1.5 \%$ ).

The per-process-domain restriction can also be interpreted as having a ruleset that describes which ports from which machines can communicate with which ports of the other machines. It is a very successful way to increase the survivability of the system, and therefore should be implemented. However, it comes with a price, as it limits the developers by forcing them to allocate fixed port numbers, and also might limit the usability of the 
machines for purposes other than the PSQ functionalities.

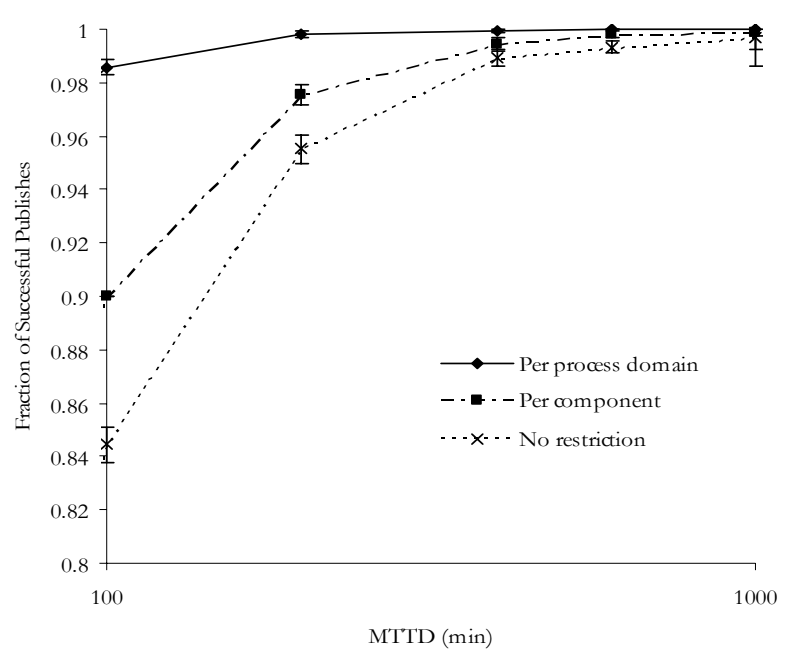

Fig. 10 - $p_{1,1}$ versus MTTD: ADF Policy Study

\section{CONCLUSION}

The complexity of emerging survivable systems, especially those that make use of multiple security approaches and technologies, calls for an integrated approach to survivability validation. We have presented the validation of a survivable publishsubscribe system using a top-down approach that begins with a precise formulation of a specific survivability requirement, and then systematically decomposes the problem into manageable tasks. As a part of the procedure, stochastic models of the system and of the attacker were presented. We conducted model-based experiments that evaluated the survivability of the system when stressed by various types of attacks by measuring the probability of success for the transactions between the clients and the core. The results show that if the average time between discoveries of new vulnerabilities is longer than one day, more than $95 \%$ of the publishes are processed correctly. The system model was used to study design trade-offs, one of which was that OS diversity in the design significantly improved the performance. Another design trade-off became apparent when we compared three ADF NIC policies: a per-process-domain policy leads to the highest availability, but constrains developers.

We described a sophisticated attacker behavior model that has wide applicability when using probabilistic modeling techniques for evaluating large networked information systems.

The integrated validation procedure (IVP) used in this work provided a collaborative environment in which a team of individuals with varied areas of expertise was able to work efficiently and optimally to produce an assurance argument that was convincing to the accreditors of the above effort. The IVP gave the designers of the system several insights into the relative merits of different protection trade-offs by comparing various algorithms, features, or infrastructures. The IVP also brought out hidden assumptions and residual requirements that forced the designers to consider issues that they might otherwise have overlooked. We hope the outlined procedure can be used by other security researchers and practitioners to validate similar survivable systems with respect to high-level quantitative survivability requirements.

The work described in this paper is an instance of an evolving validation methodology. In the future, we plan to use the IVP to validate other intrusiontolerant systems to ascertain and further refine its generic applicability, using an even wider array of evaluation techniques as the building blocks of the assurance argument. We also intend to explore avenues for further automation, such as in the decomposition of requirements, identification of appropriate assumptions, and presentation of the completed argument.

\section{Acknowledgments}

This research was supported by DARPA contract number F30602-02-C-0134. We would like to thank Franklin Webber and the other members of the DPASA team for the development of the system design and their contribution to the attack model. We would like to thank Steve Dawson, Joshua Levy, and Robert A. Riemenschneider at SRI International and Charles Payne at Adventium Labs for their work on the logical arguments. We would like to thank our colleagues at DARPA, particularly Jay Lala and Lee Badger, and at AFRL, particularly Patrick Hurley, for their constructive comments and support of this work. We would also like to thank Jenny Applequist for her editorial assistance.

\section{REFERENCES}

[1] R. J. Ellison, D. A. Fisher, R. C. Linger, H. F. Lipson, T. Longstaff, and N. R. Mead. Survivable Network Systems: An Emerging Discipline. Technical Report CMU/SEI-97TR-013, CMU Software Engineering Institute, November 1997.

[2] M. Cukier, J. Lyons, P. Pandey, H. V. Ramasamy, W. H. Sanders, P. Pal, F. Webber, R. Schantz, J. Loyall, R. Watro, M. Atighetchi, and J. Gossett. Intrusion Tolerance Approaches in ITUA. In Supplement of the 2001 International Conference on Dependable Systems and Networks, pages B-64-B-65, Göteborg, Sweden, July 2001. 
[3] Y. Deswarte, L. Blain, and J. C. Fabre. Intrusion Tolerance in Distributed Computing Systems. In Proceedings of the IEEE Symposium on Research in Security and Privacy, pages 110-121, May 1991.

[4] B. Dutertre, V. Crettaz, and V. Stavridou. Intrusion-Tolerant Enclaves. In Proceedings of the IEEE International Symposium on Security and Privacy, pages 216-224, Oakland, CA, May 2002.

[5] US Department of Defense Trusted Computer System Evaluation Criteria ("Orange Book"). http://www.radium.ncsc.mil/tpep/library/ rainbow/5200.28-STD.html, December 1985. DoD 5200.28-STD.

[6] C. Landwehr. Formal Models for Computer Security. Computer Surveys, 13(3):247-278, September 1981.

[7] J. Lowry. An Initial Foray into Understanding Adversary Planning and Courses of Action. In Proceedings of the DARPA Information Survivability Conference and Exposition II (DISCEX'01), pages 123-133, 2001.

[8] B. Littlewood, S. Brocklehurst, N. Fenton, P. Mellor, S. Page, D. Wright, J. Doboson, J. McDermid, and D. Gollmann. Towards Operational Measures of Computer Security. Journal of Computer Security, 2(2-3):211-229, 1993.

[9] E. Jonsson and T. Olovsson. A Quantitative Model of the Security Intrusion Process Based on Attacker Behavior. IEEE Transactions on Software Engineering, 23(4):235-245, April 1997.

[10] B. B. Madan, K. Goševa-Popstojanova, K. Vaidyanathan, and K. S. Trivedi. Modeling and Quantification of Security Attributes of Software Systems. In Proceedings of the 2002 International Conference on Dependable Systems and Networks (DSN 2002), pages 505514, June 2002.

[11] O. Sheyner, J. Haines, S. Jha， R. Lippmann, and J. Wing. Automated Generation and Analysis of Attack Graphs. In Proceedings of the 2002 IEEE Symposium on Security and Privacy, pages 273-284, May 2002.

[12] R. Ortalo, Y. Deswarte, and M. Kaâniche. Experimenting with Quantitative Evaluation Tools for Monitoring Operational Security. IEEE Transactions on Software Engineering, 25(5):633-650, 1999.

[13] S. Singh, M. Cukier, and W. H. Sanders. Probabilistic Validation of an IntrusionTolerant Replication System. In Proceedings of the 2003 International Conference on Dependable Systems and Networking (DSN-
2003), pages 615-624, San Francisco, CA, 2003.

[14] V. Gupta, V. Lam, H. V. Ramasamy, W. H. Sanders, and S. Singh. Dependability and Performance Evaluation of Intrusion-Tolerant Server Architectures. In Proceedings of LADC 2003: The 1st Latin American Symposium on Dependable Computing, Lecture Notes in Computer Science, volume 2847, pages 81101, São Paulo, Brazil, October 2003.

[15] P. G. Bishop and R. E. Bloomfield. The SHIP Safety Case Approach. In Proceedings of the 1995 IFAC Conference on Computer Safety, Reliability and Security (SafeComp95), pages 437-451, Belgirate, Italy, October 1995.

[16] M. Abadi and L. Lamport. Conjoining Specifications. Technical Report 118, Digital Equipment Corporation Systems Research Center, December 1993.

[17] L. Lamport. Temporal Logic of Actions. ACM Transactions on Programming Languages and Systems, 16(3):872-923, May 1994.

[18] N. Shankar. A Lazy Approach to Compositional Verification. Technical Report TSL-93-08, SRI International, 1993.

[19] J. F. Meyer, A. Movaghar, and W. H. Sanders. Stochastic Activity Networks: Structure, Behavior, and Application. In Proceedings of the International Conference on Timed Petri Nets, pages 106-115, Torino, Italy, July 1985.

[20] W. H. Sanders. CDR validation report. Technical Report CDRL A007-R2, BBN Tehnologies, 2003.

[21] D. D. Deavours, G. Clark, T. Courtney, D. Daly, S. Derisavi, J. M. Doyle, W. H. Sanders, and P. G. Webster. The Möbius Framework and Its Implementation. IEEE Transactions on Software Engineering, 28(10):956-969, October 2002.

[22] Fabrice Stevens. Validation of an IntrusionTolerant Information System Using Probabilistic Modeling. Master's thesis, University of Illinois at Urbana-Champaign, 2004.

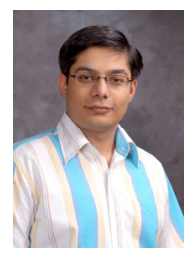

SANKALP SINGH is a Ph.D. student in the Department of Computer Science at the University of Illinois, UrbanaChampaign. His research interests include security quantification and validation. He received his $B$.Tech. in Computer Science and Engineering from the Indian Institute of Technology, Kanpur in 2001 and his M.S. in Computer Science from the University of Illinois in 2003. His email address is sankalps@crhc.uiuc.edu. 


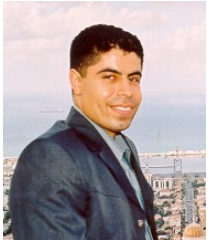

ADNAN AGBARIA received his B.A, M.A, and Ph.D. degrees in 1994, 1997, and 2002 respectively. Both the B.A. and M.A. are in Mathematics and Computer Science, from Haifa University, and the Ph.D. is in computer science from Technion - Israel Institute of Technology. Currently, he works as a postdoctoral research associate in the Coordinated Science Laboratory, University of Illinois at UrbanaChampaign. His research interests include faulttolerant and intrusion-tolerant computing, network security, parallel and distributed computing, and computer networking. He is also a member of the ACM and IEEE. His email address is adnan@crhc.uiuc.edu.

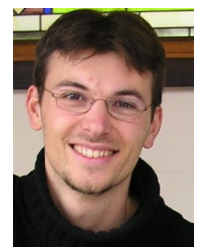

FABRICE STEVENS received his B.S. in Computer Engineering from ENST (Ecole Nationale Superieure des Telecommunications, Paris, France) in 2002, and his M.S. in Computer Engineering from the University of Illinois at Urbana-Champaign in 2004. He is currently working on network security at France Telecom Research and Development. His email address is fabrice.stevens@francetelecom.com.

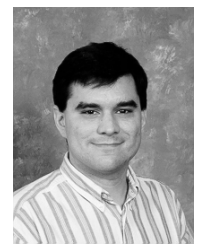

TOD COURTNEY received his Bachelor degree in Computer Engineering in 1994 and his Masters in Electrical Engineering in 1996, both from the University of Illinois. He is currently a researcher at the University of Illinois. His interests include performance, dependability, and security system evaluation, as well as stochastic modeling algorithms and software. His email address is tod@crhc.uiuc.edu.

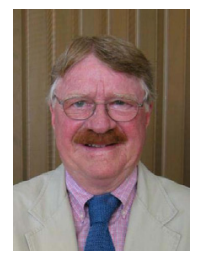

JOHN F. MEYER is a Professor Emeritus in the Department of Electrical Engineering and Computer Science at the University of Michigan, Ann Arbor, Michigan. He has been active in computer and systems research for over 45 years, resulting in a wide variety of publications concerning the areas of faulttolerant computing and system evaluation. He joined the Michigan faculty in 1967 and, in addition to his university appointments, has held visiting research appointments at laboratories in England, France, Italy, Japan, and Sweden. Prior to 1967, he was a Research Engineer at the California Institute of Technology Jet Propulsion Laboratory, where his contributions included the first patent issued to the National Aeronautics and Space Administration. His current research interests concern the development and application of models for evaluating the performability and survivability of distributed computer and communication systems. He is a Life Fellow of the IEEE and has served the IEEE Computer Society in various capacities, including Chair of the Technical Committee on Fault-Tolerant
Computing and membership on the Society's Board of Governors. He is also active in IFIP and has received the Silver Core from their General Assembly for his services to Working Group 10.4. His email address is jfm@eecs.umich.edu.

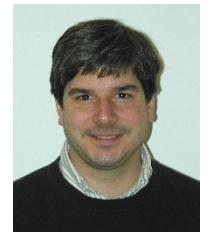

WILLIAM H. SANDERS is a Donald Bigger Willett Professor of Engineering in the Department of Electrical and Computer Engineering and the Coordinated Science Laboratory at the University of Illinois. $\mathrm{He}$ is the Director of the Information Trust Institute (ITI) at the University of Illinois. He is serving as the Vice-Chair of IFIP Working Group 10.4 on Dependable Computing. In addition, he serves on the editorial board of Performance Evaluation and is the Area Editor for Simulation and Modeling of Computer Systems for the ACM Transactions on Modeling and Computer Simulation. He is a past Chair of the IEEE Technical Committee on FaultTolerant Computing. He is a Fellow of the IEEE and the ACM. Dr. Sanders's research interests include performance/dependability evaluation, dependable computing, and reliable distributed systems. He has published more than 160 technical papers in those areas. He served as the General Chair of the 2003 Illinois International Multiconference on Measurement, Modelling, and Evaluation of Computer-Communication Systems. He has served as co-Chair of the program committees of the 29th International Symposium on Fault-Tolerant Computing (FTCS-29), the Sixth IFIP Working Conference on Dependable Computing for Critical Applications, Sigmetrics 2003, PNPM 2003, and Performance Tools 2003, and has served on the program committees of numerous conferences and workshops. He is a co-developer of three tools for assessing the performability of systems represented as stochastic activity networks: METASAN, UltraSAN, and Möbius. Möbius and UltraSAN have been distributed widely to industry and academia; more than 300 licenses for the tools have been issued to universities, companies, and NASA for evaluating the performance, dependability, security, and performability of a variety of systems. He is also a co-developer of the Loki distributed system fault injector and the AQUA/ITUA middlewares for providing dependability/security to distributed and networked applications. His email address is whs@crhc.uiuc.edu.

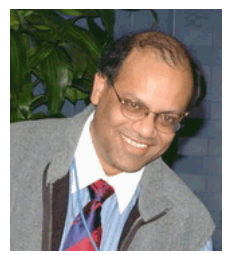

PARTHA PAL is a division scientist at BBN Technlogies, and he leads the survivability research thrust in the Distributed Systems Advanced Middleware Technology group. He is currently working on the architecture, design, implementation, and evaluation of a pathfinder system for the U.S. Dept. of Defense. He is a senior member of the IEEE. His email address is ppal@bbn.com. 